\title{
Temperature dependence of electrical conductivity and dilepton rates from hot quenched lattice QCD
}

\author{
Olaf Kaczmarek* and Marcel Müller \\ Fakultät für Physik, Universität Bielefeld, D-33615 Bielefeld, Germany \\ E-mail: okacz@physik.uni-bielefeld.de
}

\begin{abstract}
We present new results on the continuum extrapolation of the vector current correlation function in the deconfined phase for three temperatures close to the critical temperature utilizing quenched clover improved Wilson fermions and light quark masses. A systematic analysis on multiple lattice spacing allows to perform the continuum limit of the correlation function and to extract spectral properties in the continuum limit. These results provide constraints for the electrical conductivity and the thermal dilepton rates in the quark gluon plasma for the given temperature range. In addition results on the continuum extrapolation at finite momenta related to thermal photon rates are presented.
\end{abstract}

31st International Symposium on Lattice Field Theory - LATTICE 2013

July 29 - August 3, 2013

Mainz, Germany

\footnotetext{
${ }^{*}$ Speaker.
} 

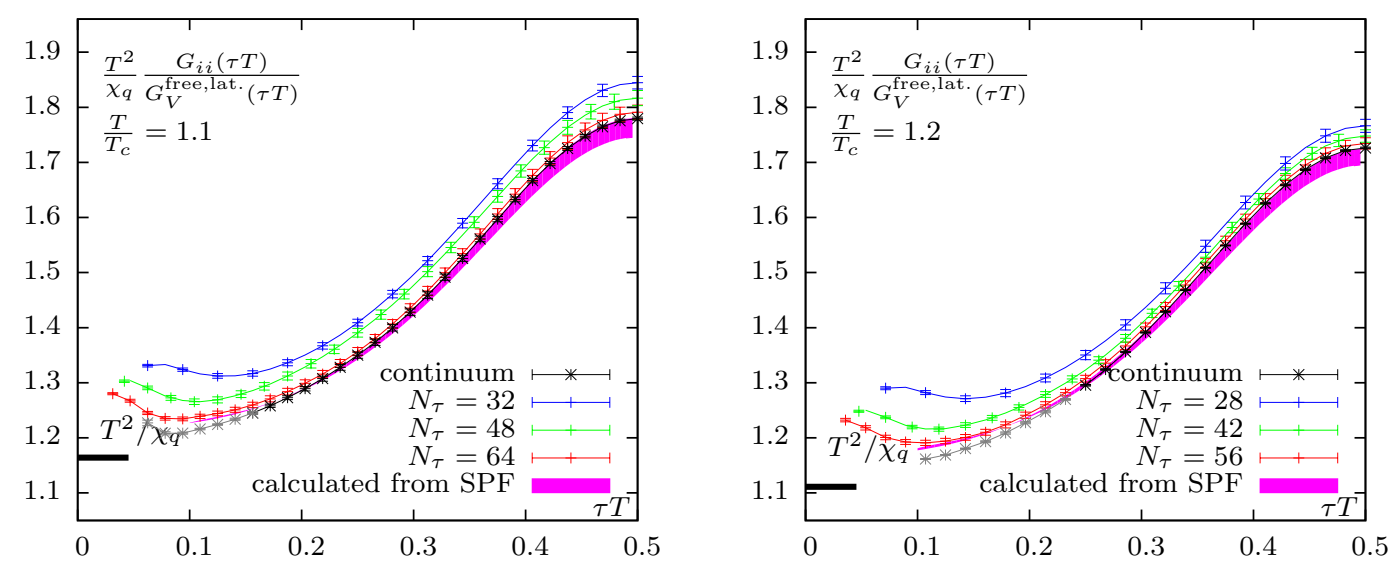

Figure 1: Vector meson correlation function $G_{i i}(\tau T)$ at $1.1 T_{c}$ (left) and $1.2 T_{c}$ (right) for the three lattice spacings and the continuum extrapolation. The band shows the result and systematic error of the fit using our Ansatz for the spectral function.

\section{Introduction}

Thermally produced dileptons and photons are important experimental observables to study the the quark gluon plasma medium produced in current heavy-ion-experiments at LHC and RHIC [1]. Both quantities are related to the vector meson spectral function and therefore indirectly to the vector correlation function. The energy regime of the current experimental studies requires non-perturbative ab initio lattice QCD calculations. The results presented in this article extend our previous studies $[2,3,4]$ where we performed the continuum extrapolation of the vector meson correlation function for the first time at a temperature of $T / T_{c}=1.45$ in the quenched approximation using non-perturbatively clover improved Wilson fermions. Here we will discuss the temperature dependence of the electrical conductivity and dilepton rates obtained from continuum extrapolated correlation functions at temperatures of 1.1, 1.2 and 1.4 $T_{c}$.

\section{Vector correlation function}

The Euclidean time two-point correlation function $G(\tau, \vec{p})$ of the vector current $J_{\mu}$ is a quantity directly accessible in lattice QCD calculations,

$$
G_{\mu v}(\tau, \vec{p})=\int d^{3} x J_{\mu}(\tau, \vec{x}) J_{v}^{\dagger}(0, \overrightarrow{0}) e^{i \vec{p} \vec{x}} \quad \text { with } \quad J_{\mu}(\tau, \vec{x})=\bar{q}(\tau, \vec{x}) \gamma_{\mu} q(\tau, \vec{x}) .
$$

Only contributions of quark line connected diagrams are included. Disconnected diagrams cause a high numerical effort and are expected to be small in the high temperature phase of QCD $[5,6]$. The correlation function directly relates to the spectral function via

$$
G_{H}(\tau, \vec{p}, T)=\int_{0}^{\infty} \frac{d \omega}{2 \pi} \rho_{H}(\omega, \vec{p}, T) \frac{\cosh (\omega(\tau-1 / 2 T))}{\sinh (\omega / 2 T)} \quad \text { with: } \quad H=00, i i, V
$$



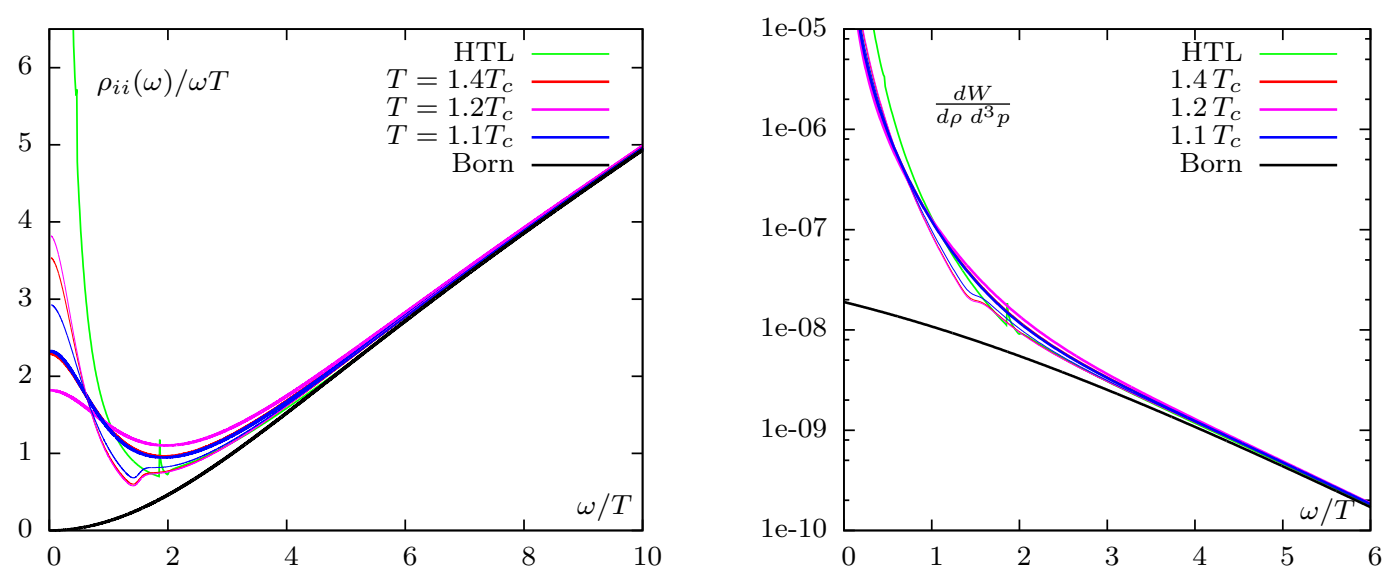

Figure 2: Results for the spectral functions (left) and thermal dilepton rates (right) calculated from the fit results to our spectral function Ansatz for three temperatures.

Here $\rho_{i i}$ denotes a sum over the spatial components and $\rho_{00}$ denotes the time-like components which is related to the quark number susceptibility. The full vector spectral function is denoted by $\rho_{V}=\rho_{00}+\rho_{i i}$.

\subsection{Ansatz for the spectral function}

The time-like component of the vector correlator $G_{00}$ and thereby the corresponding spectral function $\rho_{00}$ is related to the quark number susceptibility $\chi_{q}$. Since the quark number is conserved, the correlator is constant in (here Euclidean) time, $G_{00}(\tau T)=-\chi_{q} T$ and its spectral representation is given by a delta function

$$
\rho_{00}(\omega)=-2 \pi \chi_{q} \omega \delta(\omega) .
$$

The spatial components of the spectral functions increase quadratically for large values of $\omega$, in the free field limit for massless quarks to

$$
\rho_{i i}^{\text {free }}(\omega)=2 \pi T^{2} \omega \delta(\omega)+\frac{3}{2 \pi} \omega^{2} \tanh (\omega / 4 T) .
$$

In this limit, the delta peak in the spatial and the time-like component of the spectral function cancel. However with interactions this is not the case. The time-like component maintains a delta peak since it is linked to the conserved current, but in the spatial component the delta peak is smeared out and expected to be described by a Breit-Wigner peak $[7,8,9,10]$,

$$
\rho_{i i}^{\text {interac. }}(\omega)=\chi_{q} c_{\mathrm{BW}} \frac{\omega \Gamma}{\omega^{2}+(\Gamma / 2)^{2}}+(1+\kappa) \frac{3}{2 \pi} \omega^{2} \tanh (\omega / 4 T) .
$$

This phenomenologically inspired Ansatz leaves three parameters, the strength $\left(c_{\mathrm{BW}}\right)$ and width $(\Gamma)$ of the Breit-Wigner peak as well as $\kappa$, which accounts for the deviation from free theory (see $[11,12]$ for similar Ansätze). The relation of this Ansatz to the correlator obtained on the lattice is given by (2.2). 


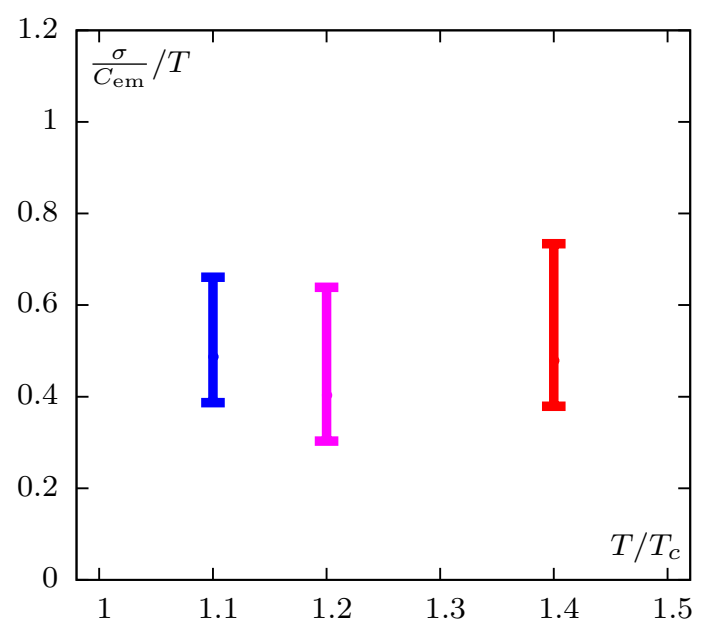

Figure 3: Temperature dependence of the electrical conductivity. Errors are obtained from the systematic error analysis (for details see [2]).

The fits are not performed directly to the correlation function $G_{i i}$ but the ratio of the correlation function normalized by the quark number susceptibility (as given in (2.3)) to become independent of any renormalization and by the free field correlation function $G_{V}^{\text {free }}(\tau T)$, yielding a smooth function that does not fall off over multiple decades like the correlation function. Furthermore, due to asymptotic freedom, the correlation function should approach the non-interacting limit at asymptotically small distances. The spectral function is thereby fitted to reproduce

$$
\frac{G_{i i}(\tau T) / G_{00}}{G_{V}^{\text {free }}(\tau T) / G_{00}^{\text {free }}}
$$

\begin{tabular}{|c|c|c|c|c|c|c|c|}
\hline$T / T_{c}$ & $N_{\tau}$ & $N_{\sigma}$ & $\beta$ & $\kappa$ & $1 / a[\mathrm{GeV}]$ & $a[\mathrm{fm}]$ & \#conf \\
\hline \multirow{4}{*}{1.1} & 32 & 96 & 7.192 & 0.13440 & 10.4 & 0.019 & 314 \\
& 48 & 144 & 7.544 & 0.13383 & 15.5 & 0.013 & 367 \\
& 64 & 192 & 7.793 & 0.13345 & 20.4 & 0.010 & 242 \\
\hline \multirow{4}{*}{1.2} & 28 & 96 & 7.192 & 0.13440 & 10.4 & 0.019 & 232 \\
& 42 & 144 & 7.544 & 0.13382 & 15.5 & 0.013 & 417 \\
& 56 & 192 & 7.793 & 0.13345 & 20.4 & 0.010 & 195 \\
\hline \multirow{4}{*}{1.4} & 24 & 128 & 7.192 & 0.13440 & 10.4 & 0.019 & 232 \\
& 32 & 128 & 7.458 & 0.13383 & 14.1 & 0.014 & 417 \\
& 48 & 128 & 7.793 & 0.13340 & 20.4 & 0.010 & 195 \\
\hline
\end{tabular}

Table 1: Summary of simulation parameters

More details on the fit procedure and the error analysis can be found in [2]. Having obtained the spectral function, relevant properties of the medium can be calculated, e.g. the electrical conductivity as 

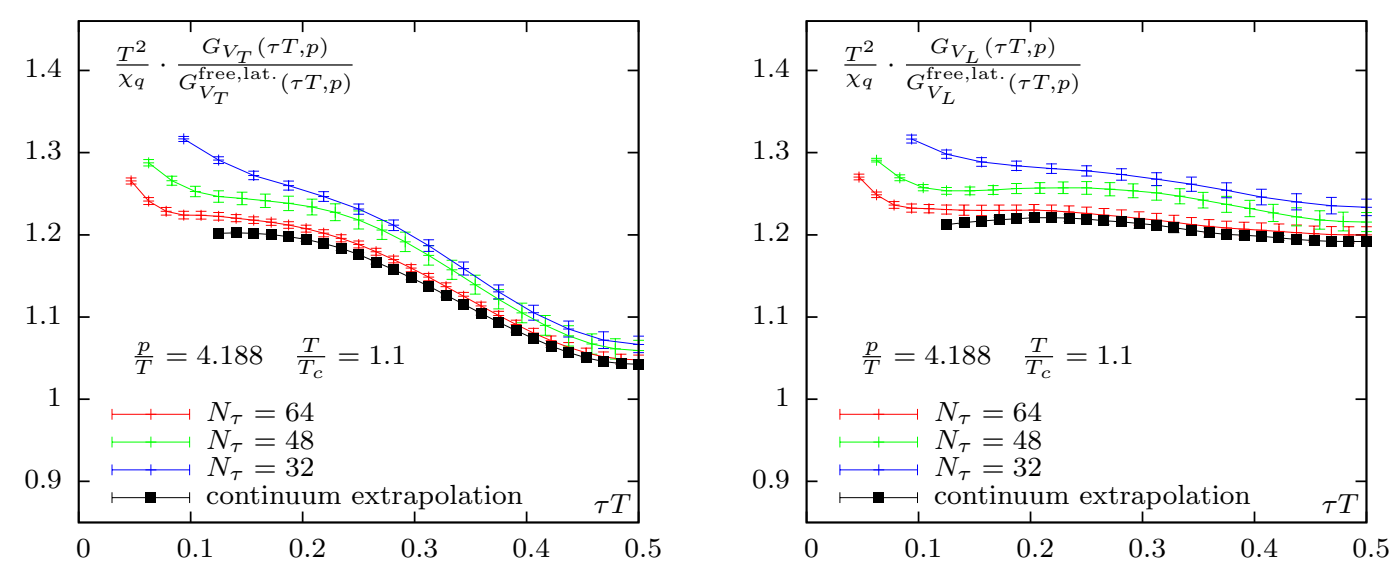

Figure 4: Transverse (left) and longitudinal (right) component of the vector correlation function at $1.1 T_{c}$ and momentum $p / T=4.188$.

$$
\frac{\sigma}{T}=\frac{C_{\mathrm{em}}}{6} \lim _{\omega \rightarrow 0} \frac{\rho_{i i}(\omega)}{\omega T} \rightarrow \sigma(T) / C_{\mathrm{em}}=2 \chi_{q} c_{\mathrm{BW}} /(3 \Gamma)
$$

where $C_{\mathrm{em}}$ is given by the elementary charges $Q$ of the quark flavor $f$ as $C_{\mathrm{em}}=\sum_{f} Q_{f}^{2}$, and the thermal production rate of dilepton pairs as

$$
\frac{d W}{d \omega d^{3} p}=\frac{5 \alpha^{2}}{54 \pi^{3}} \frac{1}{\omega^{2}\left(e^{\omega / T}-1\right)} \rho_{i i}(\omega, p, T) .
$$

\section{Results for correlators and spectral function fits}

We have calculated vector correlation functions using non-perturbatively clover-improved Wilson fermion on a set of quenched gauge field configurations stated in Tab. 1. For each temperature three lattice spacings were used in order to perform the continuum limit. While the results of [2] were obtained on a fixed spatial lattice of $N_{\sigma}=128$, recent results were calculated at fixed aspect ratio of $N_{\sigma} / N_{\tau}=3.00$ for $1.1 T_{c}$ and $N_{\sigma} / N_{\tau}=3.43$ at $1.2 T_{c}$ and therefore at a fixed physical volume. This set-up allows for a continuum extrapolation also at finite momenta for the two lowest temperatures. In Fig. 1 the results of the vector correlation function $G_{i i}(\tau T)$ for $1.1 T_{c}$ (left) and $1.2 T_{c}$ (right) are shown together with the corresponding continuum extrapolations. All results are normalized by the free non-interacting correlation function, $G_{V}^{\text {free,lat }}(\tau T)$, and with the quark number susceptibility, $\chi_{q}$, to remove any renormalization constants. While the results on finite lattices show strong cut-off effects at all distances and a wrong rising behavior towards small distances, the continuum extrapolations are well behaved down to distances around 0.1 $\tau T$. The band shows the result and systematic error of the fit using the Ansatz (2.5). The rise of these ratios towards the mid-point can be identified as a first indication for a transport contribution.

The resulting spectral functions using the Ansatz (2.5) are shown in Fig. 2 (left) and the dilepton rates obtained from (2.8) in Fig. 2 (right). Both results are compared to a dilepton spectrum calculated within the hard thermal loop approximation [13]. In the limit of vanishing $\omega$ one can 

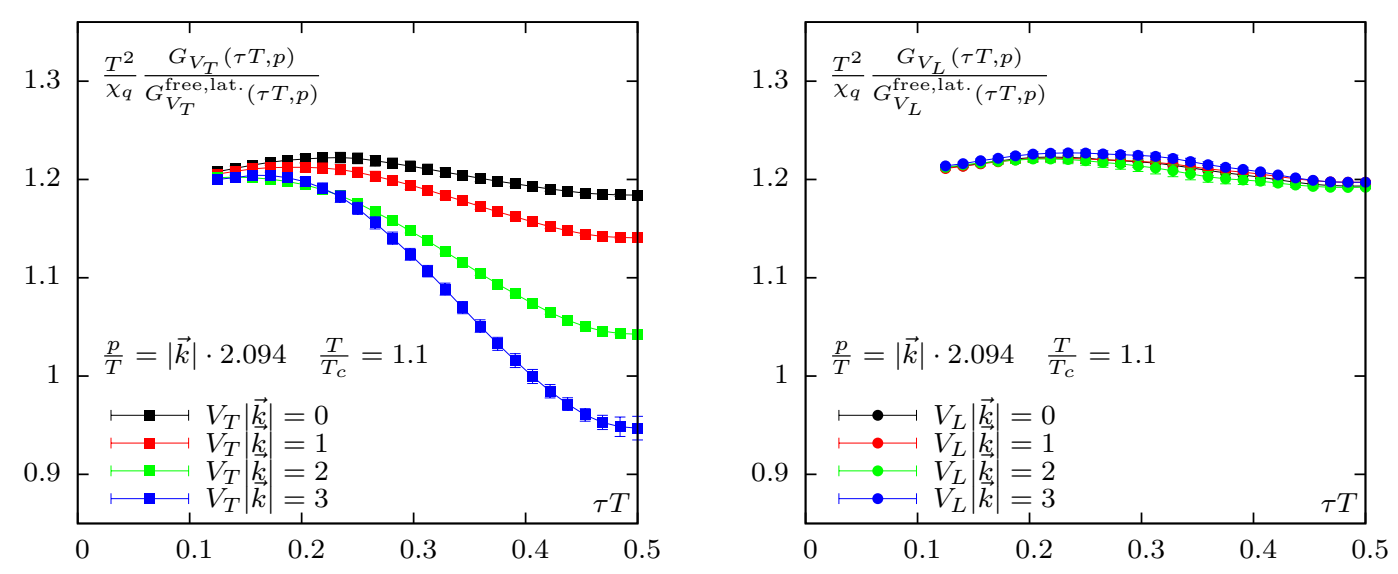

Figure 5: Continuum extrapolated vector correlation function in transversal (left) and longitudinal (right) polarization $1.1 T_{c}$ and four momenta.

read of the electrical conductivity (2.7) from Fig. 2 (left). The results for $\sigma / T$ plotted in Fig. 3 show no temperature dependence in the analyzed T-range within the systematic errors. For a comparison with results from calculations with dynamical quarks on finite lattices see [14, 15].

\section{Vector correlation function at non-vanishing momenta}

For the two lowest temperatures, $1.1 T_{c}$ and 1.2 $T_{c}$, the vector correlation functions for the three lattice spacings were calculated at a fixed aspect ratio of $N_{\sigma} / N_{\tau}$. This allows to perform the continuum extrapolation also for non-vanishing momenta, $\vec{p}$. In Fig. 4 and Fig. 5 the results for the vector correlation functions in the transversal (left) and longitudinal (right) polarization channel at a temperature of $1.1 T_{c}$ are shown. While for the longitudinal channel no momentum dependence is observed within the errors, the transversal channel shows a pronounced $\vec{p}$-dependence which is weak at small separations but becomes stronger at larger distances towards the mid-point.

This behavior can qualitatively be interpreted in the context of the small-frequency behavior of the two channels in the leading-log Boltzmann approximation [10]. In the limit of $\omega \rightarrow 0$ the transverse spectral function $\rho_{T}(\omega, \vec{p}) / \omega$ is non-vanishing for all momenta $\vec{p}$, while it is zero in the longitudinal polarization, $\rho_{T}(\omega, \vec{p}) / \omega$, for all non-vanishing momenta. This leads to a different $\vec{p}$-dependence of the low-frequency region which results in a different dependence on momenta of the correlation functions at large distances and a more pronounced $\vec{p}$-dependence in the transversal channel. Due to asymptotic freedom one expects that both channels approach each other at small distances. Based on these observations, the different behavior in the transversal and longitudinal polarization can qualitatively be understood, but for a more detailed understanding the spectral properties need to be determined in the future. For a comparison of our continuum extrapolated vector correlation function at non-vanishing momenta with a perturbative NLO calculation see [16]. 


\section{Conclusion}

Continuum results for the vector current correlation functions in the temperature ranges $1.1 \leq$ $T / T_{c} \leq 1.4 T_{c}$ were presented. Using a rather simple, phenomenologically inspired Ansatz for the vector spectral function and a careful analysis of the systematic uncertainties, this sheds light into the temperature dependence of the electrical conductivity and dilepton rates in an energy regime currently accessible in heavy-ion experiments. The continuum extrapolated vector correlation function at non-vanishing momenta show a qualitatively different behavior in the transversal and longitudinal polarization. The results allow for study of the spectral properties and determination of the photon rates in the future.

\section{Acknowledgment}

The results have been achieved by using the PRACE Research Infrastructure resource JUGENE based at the Jülich Supercomputing Centre in Germany and the Bielefeld GPU-cluster resources. This work has been partly supported by the IRTG/GRK 881 "Quantum Fields and Strongly Interacting Matter".

\section{References}

[1] R. Rapp, J. Wambach, and H. van Hees, The Chiral Restoration Transition of QCD and Low Mass Dileptons, in Landolt-Börnstein, vol. I-23, 4-1. Springer-Verlag, 2010. arXiv:0 901.3289.

[2] H.-T. Ding, A. Francis, O. Kaczmarek, F. Karsch, E. Laermann, et al., Phys.Rev. D83 (2011) 034504.

[3] A. Francis and O. Kaczmarek, Prog.Part.Nucl.Phys. 67 (2012) 212.

[4] O. Kaczmarek et al., PoS ConfinementX (2012) 185.

[5] C. Allton, M. Doring, S. Ejiri, S. Hands, O. Kaczmarek, et al., Phys.Rev. D71 (2005) 054508.

[6] R. V. Gavai, S. Gupta, and P. Majumdar, Phys.Rev. D65 (2002) 054506.

[7] G. Aarts and J. M. Martinez Resco, JHEP 0204 (2002) 053.

[8] G. D. Moore and J.-M. Robert, hep-ph/0607172.

[9] P. Petreczky and D. Teaney, Phys.Rev. D73 (2006) 014508.

[10] J. Hong and D. Teaney, Phys.Rev. C82 (2010) 044908.

[11] Y. Burnier and M. Laine, Eur.Phys.J. C72 (2012) 1902.

[12] R. Rapp, arXiv:1304.2309 [hep-ph].

[13] E. Braaten and R. D. Pisarski, Nucl.Phys. B337 (1990) 569.

[14] A. Amato et al., Phys. Rev. Lett. 111, 172001 (2013).

[15] B. B. Brandt, A. Francis, H. B. Meyer and H. Wittig, JHEP 1303, 100 (2013).

[16] M. Laine, JHEP 1311, 120 (2013). 